\title{
Improved Pharmacokinetics with BAY 81-8973 Versus Antihemophilic Factor (Recombinant) Plasma/Albumin-Free Method: A Randomized Pharmacokinetic Study in Patients with Severe Hemophilia A
}

\author{
Anita Shah $^{1}$ (D) - Alexander Solms ${ }^{2} \cdot$ Dirk Garmann $^{3} \cdot$ Yvonne Katterle $^{2}$ • \\ Verzhiniya Avramova ${ }^{4} \cdot$ Stanislav Simeonov $^{4} \cdot$ Toshko Lissitchkov $^{4}$
}

Published online: 22 December 2016

(c) The Author(s) 2016. This article is published with open access at Springerlink.com

\begin{abstract}
Background BAY 81-8973 is a full-length, unmodified, recombinant human factor VIII (FVIII) for the treatment of hemophilia A.

Objective The aim of this study was to compare the pharmacokinetic (PK) profile of BAY 81-8973 with antihemophilic factor (recombinant) plasma/albumin-free method (rAHF-PFM)

Patients/Methods In this phase I, open-label, crossover study, men aged 18-65 years with severe hemophilia A and $\geq 150$ exposure days to FVIII were randomized to receive a single intravenous infusion of $50 \mathrm{IU} / \mathrm{kg} \mathrm{BAY}$ 81-8973 or rAHF-PFM, followed by crossover to a single infusion of the other treatment. FVIII levels were measured
\end{abstract}

A. Shah and A. Solms contributed equally to this work.

Anita Shah

anita.shah@bayer.com

Bayer, 100 Bayer Blvd, Whippany, NJ 07981, USA

Bayer AG, Berlin, Germany

Bayer AG, Wuppertal, Germany

4 Specialized Hospital for Active Treatment of Hematologic Diseases, Sofia, Bulgaria in plasma over $48 \mathrm{~h}$ using one-stage and chromogenic assays. PK parameters, including area under the curve from time zero to the last data point $\left(\mathrm{AUC}_{\text {last}}\right.$; primary outcome) and half-life $\left(t_{1 / 2}\right)$ were calculated. A population PK model was developed to simulate various treatment scenarios. Results Eighteen patients were randomized and analyzed. Using both assays, geometric mean (coefficient of variation [\%CV]) $\mathrm{AUC}_{\text {last }}$ was significantly higher, and $t_{1 / 2}$ was significantly longer, for BAY 81-8973 versus rAHF-PFM (one-stage, $\mathrm{AUC}_{\text {last }}: 1660 \mathrm{IU} \cdot \mathrm{h} / \mathrm{dL}$ [29.4] vs. $1310 \mathrm{IU} \cdot \mathrm{h} / \mathrm{dL}$ [29.0], $p<0.0001$; one-stage, $t_{1 / 2}: 14.5$ [25.7] vs. $11.7 \mathrm{~h}$ [27.3], $p<0.0001)$. Simulations showed that median time to $1 \mathrm{IU} / \mathrm{dL}$ was approximately $27 \%$ longer for BAY 81-8973 versus rAHF-PFM over doses of $25-50 \mathrm{IU} / \mathrm{kg}$; plasma levels $>1 \mathrm{IU} / \mathrm{dL}$ could be maintained with $14.4 \mathrm{IU} / \mathrm{kg}$ BAY $81-8973$ or $39.1 \mathrm{IU} / \mathrm{kg}$ rAHF-PFM $3 \times /$ week.

Conclusions BAY 81-8973 showed a superior PK profile versus rAHF-PFM. The same FVIII trough threshold level could be achieved with lower doses of BAY 81-8973 versus rAHF-PFM.

ClinicalTrials.gov: NCT02483208. 


\section{Key Points}

Using a randomized crossover study design in patients with severe hemophilia A, a single infusion of BAY 81-8973 $\left(\right.$ Kovaltry $^{\circledR}$ ) was associated with significantly improved area under the curve, halflife, mean residence time, and lower clearance compared with a single infusion of antihemophilic factor (recombinant) plasma/albumin-free method (rAHF-PFM [Advate ${ }^{\circledR}$ ]).

Simulation data using a population pharmacokinetic (PK) model indicated that a higher proportion of patients could maintain factor VIII (FVIII) levels above threshold levels of $1,3,5$, or $10 \mathrm{IU} / \mathrm{dL}$ for longer periods of time with BAY 81-8973 compared with rAHF-PFM.

FVIII products with improved PK profiles may provide additional protection against bleeding by increasing FVIII trough levels, and may allow for less frequent infusions in some patients with severe hemophilia A.

\section{Introduction}

Treatment of hemophilia A typically requires factor VIII (FVIIII) replacement therapy. In most developed countries with adequate resources, FVIII prophylaxis is standard care for patients with hemophilia A [1, 2]. However, insufficient levels of infused FVIII resulting from suboptimal adherence to prophylaxis or individual differences in FVIII pharmacokinetics (PK) may result in breakthrough bleeding during prophylaxis [3-5]. Although the appropriate level of FVIII to prevent bleeding in individual patients varies [6,7], increased time spent with low FVIII levels is considered an important determinant of breakthrough bleeding during prophylaxis [8]. Thus, maintenance of FVIII above threshold levels for long periods is perceived to be beneficial. The desired threshold level for a given patient can vary based on the bleeding phenotype, activity level, genetic mutation, and other variables. Hemophilia treaters may target threshold levels of $1,3,5$, or $10 \mathrm{IU} / \mathrm{dL}$ FVIII, or higher, while selecting a dosing regimen for prophylaxis [6-8].

BAY 81-8973 is a full-length, unmodified, recombinant human FVIII that has the same amino acid sequence as Bayer's sucrose-formulated recombinant FVIII (rFVIII-FS; Kogenate ${ }^{\circledR}$ FS, Bayer, Berkeley, CA, USA) but produced with certain, more advanced manufacturing technologies [9]. The changes and enhancements to the BAY 81-8973 manufacturing process include use of a $20-\mathrm{nm}$ viral filtration step capable of removing small non-enveloped viruses and potential protein aggregates; elimination of human- or animal-derived raw materials from the production process; and use of a new, more robust, and higher productivity cell line developed through introduction of the gene for human heat shock protein 70 [9]. Refinements to the manufacturing process of BAY 81-8973 have resulted in an rFVIII product of high and consistent purity with a consistently high degree of sialic acid capping of $\mathrm{N}$-terminal glycans [9], which may affect the half-life $\left(t_{1 / 2}\right)$ of some mammalian proteins [10]. BAY 81-8973 has an improved PK profile compared with rFVIII-FS, including increased area under the curve (AUC), $t_{1 / 2}$, and mean residence time (MRT), as well as decreased clearance (CL) [9]. The safety and efficacy of BAY 81-8973 for prophylaxis (two to three times per week) and on-demand treatment has been demonstrated in children, adolescents, and adults with severe hemophilia A [11-13]. The objective of the current study was to compare the PK profile of BAY 81-8973 with a marketed full-length rFVIII product, antihemophilic factor (recombinant) plasma/albumin-free method (rAHF-PFM). Data collected using the chromogenic assay were used to develop a population PK (popPK) model for BAY 81-8973 and rAHF-PFM to simulate various treatment scenarios in larger patient populations.

\section{Methods}

\subsection{Patients}

Men aged 18-65 years with severe hemophilia A (FVIII $<1 \mathrm{IU} / \mathrm{dL}$ [\%]) previously treated with FVIII for $\geq 150$ exposure days were eligible for inclusion in the study. Patients must have also been able to stop FVIII treatment to complete a $\geq 3$-day washout period before study entry and between treatments. Key exclusion criteria included the presence or history of an FVIII inhibitor $(\geq 0.6$ Bethesda units $/ \mathrm{mL}$ ), diagnosis of any bleeding disorders other than hemophilia A, platelet count $<75,000 / \mathrm{mm}^{3}$, serum creatinine $>2 \times$ the upper limit of normal (ULN), and alanine aminotransferase or aspartate aminotransferase $>5 \times$ the ULN.

\subsection{Study Design}

This was a phase I, single-dose, open-label, randomized, crossover study to compare the PK of BAY 81-8973 with rAHF-PFM (ClinicalTrials.gov identifier: NCT02483208). Patients $(N=18)$ were randomized according to a computer-generated randomization list to receive a single infusion of $50 \mathrm{IU} / \mathrm{kg}$ BAY 81-8973 (Kovaltry ${ }^{\circledR}$; Bayer, 
Berkeley, CA, USA) or 50 IU/kg rAHF-PFM (Advate ${ }^{\circledR}$; Baxalta, Westlake Village, CA, USA), followed by crossover to a single infusion of the other treatment, with washout periods of $\geq 3$ days before each single dose. Both products were administered as 10 -min intravenous infusions. Informed consent was provided by the patients, and the protocol was approved by the site's independent Ethics Committee/Institutional Review Board.

\subsection{Pharmacokinetic (PK) Assessments}

Plasma samples were collected predose and $0.25,0.5,1,3$, $6,8,24,30$, and $48 \mathrm{~h}$ postdose for PK assessments. FVIII levels were measured using the one-stage and chromogenic assays, both of which are commonly used for PK studies conducted using FVIII products [9, 14-17]. For the onestage assay, plasma concentrations of BAY 81-8973 and rAHF-PFM were determined by a turbidimetric assay with activated partial thromboplastin time measurement on the ACL Advance System (Instrumentation Laboratory, Bedford, MA, USA) against a calibration curve of standard human plasma. The calibration range of the procedure for BAY 81-8973 was from 10.0 IU/L (or $1 \mathrm{IU} / \mathrm{dL}$, lower limit of quantitation [LLOQ]) to $600 \mathrm{IU} / \mathrm{L}$ (or $60 \mathrm{IU} / \mathrm{dL}$, upper limit of quantification [ULOQ]). Quality control (QC) samples in the concentration range from 25.0 to $450 \mathrm{IU} / \mathrm{L}$ were determined with an accuracy of 96.0-104\% and a precision of $7.64-14.0 \%$. The calibration range of the procedure for rAHF-PFM was from 10.0 IU/L (or $1 \mathrm{IU} / \mathrm{dL}$ [LLOQ]) to $600 \mathrm{IU} / \mathrm{L}$ (or $60 \mathrm{IU} / \mathrm{dL}$ [ULOQ]). QC samples in the concentration range from 25.0 to $450 \mathrm{IU} / \mathrm{L}$ were determined with an accuracy of $98.1-112 \%$ and a precision of $6.37-10.0 \%$. For the chromogenic assay, FVIII activity of BAY 81-8973 and rAHF-PFM was determined in citrate plasma by an enzymatic activity assay with chromogenic readout using a commercially available kit and normal human plasma standard as a calibrator. The calibration range of the procedure for BAY 81-8973 was from 10.0 (LLOQ) to $500 \mathrm{IU} / \mathrm{L}$ (ULOQ). QC samples in the concentration range from 25.0 to $375 \mathrm{IU} / \mathrm{L}$ were determined with an accuracy of $94.3-106 \%$ and a precision of $3.75-7.09 \%$. The calibration range of the procedure for rAHF-PFM was from 10.0 (LLOQ) to 500 IU/L (ULOQ). QC samples in the concentration range from 25.0 to 375 IU/L were determined with an accuracy of 98.8-106\% and a precision of $2.65-4.00 \%$. For both methods, samples above the calibration range were diluted with severe hemophilic plasma into the working dilution range before analysis. Dilution linearity and parallelism of QC versus patient samples has been proven during validation of the one-stage and chromogenic assays.

The following PK parameters were assessed using data collected from both assays: AUC from time zero to the last data point ( $\mathrm{AUC}_{\text {last }}$; primary variable), AUC from time zero to infinity $\left(\mathrm{AUC}_{\mathrm{inf}}\right)$, maximum concentration $\left(C_{\max }\right)$, $t_{1 / 2}, \mathrm{CL}, \mathrm{MRT}$, and volume of distribution at steady state $\left(V_{\mathrm{ss}}\right)$. All parameters were calculated using noncompartmental methods (WinNonlin ${ }^{\circledR}$ software; Pharsight Corporation, Mountain View, CA, USA). AUC was calculated using the log-linear trapezoidal method, and $t_{1 / 2}$ was calculated using the regression analysis of the linear part of the curve on a semilogarithmic scaled plot.

\subsection{Population PK (PopPK) Model}

A popPK model for BAY 81-8973 and rAHF-PFM was developed based on data obtained by the chromogenic assay. The analysis was conducted using the nonlinear mixed-effect modeling approach as implemented in NONMEM $^{\circledR}$ (version 7, level 2.0; ICON, Hanover, MD, USA). A two-compartment model was used as the structural PK model (ADVAN5) and was parameterized by CL, volume of the central compartment $(V 1)$, intercompartmental clearance $(Q)$, and volume of the peripheral compartment (V2). In the first step of the model development process, the PK of BAY 81-8973 and rAHF-PFM were assumed to be identical. In the next step, the model was sequentially redefined by testing whether BAY 81-8973 and $\mathrm{rAHF}-\mathrm{PFM}$ have statistically significant differences in CL, $V 1, Q$, and/or $V 2$. Because of the small study size, no covariate analysis was conducted.

Individual PK parameters were derived from the popPK model and were used to estimate $t_{1 / 2}$ and predict trough levels for a dose of $30 \mathrm{IU} / \mathrm{kg}$ BAY 81-8973 and rAHFPFM administered twice weekly or three times weekly. Furthermore, the popPK model was used to simulate switching from $30 \mathrm{IU} / \mathrm{kg}$ rAHF-PFM twice weekly or three times weekly at steady state to the same dose and dosing frequency of BAY 81-8973.

\subsection{Simulations}

The popPK model was used to assess 'time to reach' and 'time below' FVIII threshold levels of 1, 3, 5, and $10 \mathrm{IU} / \mathrm{dL}$ based on a larger virtual patient population after administration of various doses of BAY 81-8973 or rAHFPFM. Therefore, steady-state profiles for a virtual population ( $n=1000$ patients) were simulated. The proportion of patients above these thresholds and the required doses to achieve these thresholds were also compared between the two products.

\subsection{Statistical Analysis}

For statistical analysis of the PK parameters obtained by noncompartmental analysis, a log-normal distribution of 
the parameters was assumed. Log-transformed parameters were analyzed using analysis of variance (ANOVA), including sequence, patient (sequence), period, and treatment effects. Based on these analyses, point estimates (least squares) means and CIs (90\% and 95\%) for the BAY 81-8973:rAHF-PFM ratio were calculated by retransformation of the logarithmic data using the intraindividual SD of the ANOVA. A sample size of 16 patients was determined to be sufficient to compare PK based on expected intraindividual coefficients of variation between $12.5 \%$ and $20 \%$ for $C_{\max }$ and AUC from previous studies with BAY 81-8973 and rFVIII-FS. The actual results from our study had intraindividual coefficients of variation of $12 \%$ and $6.8 \%$ for AUC based on the one-stage and chromogenic assays, respectively, and $10 \%$ and $4.9 \%$ for $C_{\max }$ based on the one-stage and chromogenic assays, respectively.

\section{Results}

\subsection{Patients}

A total of 18 patients from a single center in Bulgaria were randomized and received single doses of BAY 81-8973 and rAHF-PFM. Mean \pm SD age for all patients was $37.3 \pm 13.8$ years, and all patients were white. Demographic data for all 18 patients are provided in Table 1. All patients completed the study and were analyzed.

Table 1 Baseline demographics and clinical characteristics

\begin{tabular}{ll}
\hline & All patients $[N=18]$ \\
\hline Age, years & \\
Mean \pm SD & $37.3 \pm 13.8$ \\
Median (range) & $36(19-64)$ \\
Race $[n(\%)]$ & $18(100)$ \\
White & \\
Weight, kg & $75.7 \pm 13.7$ \\
Mean \pm SD & $80.0(55.0-99.0)$ \\
Median (range) & \\
BMI, kg/m ${ }^{2}$ & $24.5 \pm 3.7$ \\
Mean \pm SD & $26.1(18.5-28.9)$ \\
Median (range) & \\
VWF, \% & $123(68-236)^{\mathrm{a}}$ \\
Mean (range) & $4(22.2)$ \\
Hemophilic arthropathy $[n(\%)]$ & $14(77.8)$ \\
HCV infection $[n(\%)]$ &
\end{tabular}

$B M I$ body mass index, $H C V$ hepatitis $\mathrm{C}$ virus, $V W F$ von Willebrand factor, $S D$ standard deviation

${ }^{\text {a }}$ Data available for 17 patients

\subsection{PK Assessments}

The FVIII concentration-time profiles show that at several timepoints, FVIII levels are higher for BAY 81-8973 compared with rAHF-PFM (Fig. 1). Predose FVIII baseline values were similar in patients for both treatments. For the one-stage assay, $3 / 18$ (range $<$ LLOQ to $2.5 \mathrm{IU} / \mathrm{dL}$ ) and $2 / 18$ (range <LLOQ to $1.5 \mathrm{IU} / \mathrm{dL}$ ) patients had FVIII levels above the LLOQ for BAY 81-8973 and rAHF-PFM, respectively. For the chromogenic assay, 4/18 (range $<$ LLOQ to $2.8 \mathrm{IU} / \mathrm{dL}$ ) and $3 / 18$ (range $<$ LLOQ to $2.0 \mathrm{IU} / \mathrm{dL}$ ) patients had levels above the LLOQ for BAY 81-8973 and rAHF-PFM, respectively. AUC last was significantly higher for BAY 81-8973 compared with rAHFPFM using the one-stage $(1660$ vs. $1310 \mathrm{IU} \cdot \mathrm{h} / \mathrm{dL}$, $p<0.0001)$ and chromogenic $(2200$ vs. $1550 \mathrm{IU} \cdot \mathrm{h} / \mathrm{dL}$, $p<0.0001)$ assays. Similar results were obtained for $\mathrm{AUC}_{\mathrm{inf}}$ (Table 2). The $t_{1 / 2}$ was also significantly longer for BAY 81-8973 versus rAHF-PFM using the one-stage (14.5 vs. $11.7 \mathrm{~h}, p<0.0001)$ and chromogenic (13.9 vs. $12.0 \mathrm{~h}$, $p<0.0001$ ) assays (Table 2). Independent of the assay, MRT was significantly higher and CL was significantly lower for BAY 81-8973 compared with rAHF-PFM (all $p<0.0001$ ), while $C_{\max }$ was similar for BAY 81-8973 and rAHF-PFM (Table 2).

\subsection{PopPK Model and Simulations}

The parameter estimates of the popPK model are shown in Table 3. Visual predictive checks showed good agreement between the popPK model and the observed data. Model diagnostics (e.g. goodness-of-fit plots such as conditional weighted residuals and standard errors of parameter estimates) indicated a precise and robust estimation of all model parameters (Fig. 2). The popPK model was used to estimate $t_{1 / 2}$ and predict individual steady-state trough levels following $30 \mathrm{IU} / \mathrm{kg}$ BAY $81-8973$ or rAHF-PFM administered twice weekly or three times weekly based on individual PK parameters from the 18 patients in the randomized crossover study. In 16 of 18 patients, $t_{1 / 2}$ was longer for BAY 81-8973 versus rAHF-PFM (Fig. 3), while in the two remaining patients, $t_{1 / 2}$ was 12.7 versus $13.1 \mathrm{~h}$ and 10.0 versus $10.9 \mathrm{~h}$ for BAY 81-8973 and rAHF-PFM, respectively. Trough levels were higher in 17 patients following BAY 81-8973 administered twice weekly compared with rAHF-PFM; for three-times-weekly administration, trough levels were higher in all patients after BAY 81-8973 administration (Fig. 4). Typical concentration-time profiles showing FVIII activity 1 week before and 1 week after a simulated switch in treatment from $30 \mathrm{IU} / \mathrm{kg}$ rAHF-PFM twice weekly or three times weekly to BAY 81-8973 at the same dose and dosing frequencies are shown in Fig. 5. 
Fig. 1 FVIII level decay curves after single-dose administration of BAY 81-8973 and rAHFPFM using the a one-stage assay and $\mathbf{b}$ chromogenic assay. FVIII factor VIII, $r A H F-P F M$ antihemophilic factor (recombinant) plasma/albuminfree method a One-Stage Assay

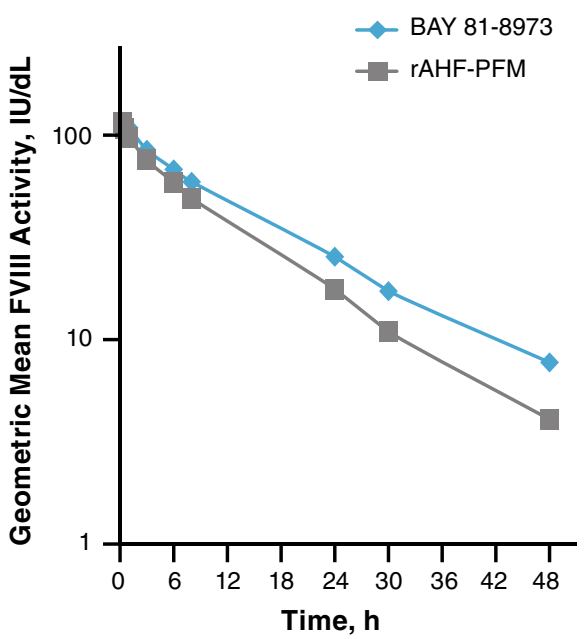

b Chromogenic Assay

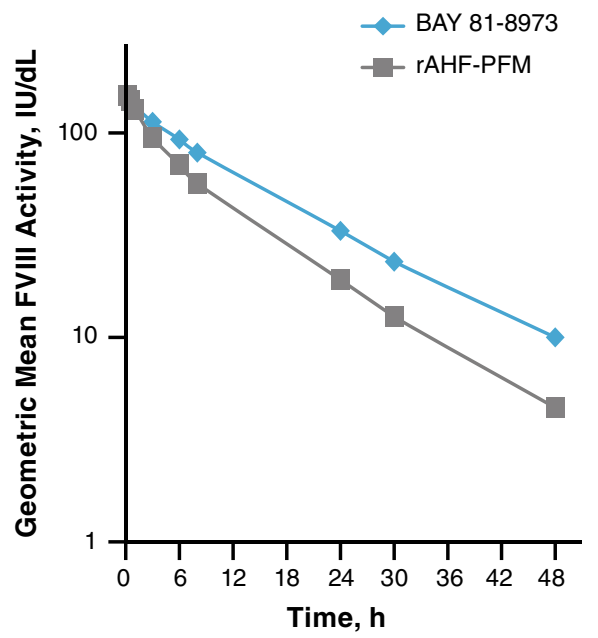

Table 2 Pharmacokinetic results following single-dose administration of BAY 81-8973 and rAHF-PFM

\begin{tabular}{|c|c|c|c|c|}
\hline & \multicolumn{2}{|c|}{ Geometric mean (\%CV) } & \multirow{2}{*}{$\begin{array}{l}\text { Geometric least squares } \\
\text { mean ratio }{ }^{a}(95 \% \mathrm{CI})\end{array}$} & \multirow[t]{2}{*}{$p$ value } \\
\hline & BAY 81-8973 & rAHF-PFM & & \\
\hline \multicolumn{5}{|l|}{ One-stage assay } \\
\hline $\mathrm{AUC}_{\text {last }}, \mathrm{IU} \cdot \mathrm{h} / \mathrm{dL}$ & $1660(29.4)$ & $1310(29.0)$ & $1.27(1.18-1.36)$ & $<0.0001$ \\
\hline $\mathrm{AUC}_{\text {inf }}, \mathrm{IU} \cdot \mathrm{h} / \mathrm{dL}$ & $1850(34.3)$ & $1400(32.4)$ & $1.32(1.21-1.44)$ & $<0.0001$ \\
\hline$C_{\max }, \mathrm{IU} / \mathrm{dL}$ & $119(15.7)$ & $116(18.5)$ & $1.02(0.95-1.10)$ & 0.51 \\
\hline$t_{1 / 2,2} \mathrm{~h}$ & $14.5(25.7)$ & $11.7(27.3)$ & $1.23(1.14-1.33)$ & $<0.0001$ \\
\hline Range & $9.65-21.4$ & $6.83-17.4$ & NA & \\
\hline MRT, h & $19.5(27.3)$ & $15.7(29.0)$ & $1.24(1.16-1.33)$ & $<0.0001$ \\
\hline $\mathrm{CL}, \mathrm{dL} / \mathrm{h} / \mathrm{kg}$ & $0.027(34.3)$ & $0.036(32.4)$ & $0.76(0.70-0.83)$ & $<0.0001$ \\
\hline$V_{\mathrm{ss}}, \mathrm{dL} / \mathrm{kg}$ & $0.53(19.4)$ & $0.56(17.3)$ & $0.94(0.88-1.00)$ & 0.07 \\
\hline \multicolumn{5}{|l|}{ Chromogenic assay } \\
\hline $\mathrm{AUC}_{\text {last }}, \mathrm{IU} \cdot \mathrm{h} / \mathrm{dL}$ & $2200(23.9)$ & $1550(27.4)$ & $1.42(1.36-1.49)$ & $<0.0001$ \\
\hline $\mathrm{AUC}_{\mathrm{inf}}, \mathrm{IU} \cdot \mathrm{h} / \mathrm{dL}$ & $2440(28.5)$ & $1650(31.0)$ & $1.48(1.41-1.55)$ & $<0.0001$ \\
\hline$C_{\max }, \mathrm{IU} / \mathrm{dL}$ & $151(19.9)$ & $153(17.1)$ & $0.98(0.95-1.02)$ & 0.32 \\
\hline$t_{1 / 2}, \mathrm{~h}$ & $13.9(25.1)$ & $12.0(23.3)$ & $1.16(1.10-1.23)$ & $<0.0001$ \\
\hline Range & $9.95-22.2$ & $9.06-17.9$ & NA & \\
\hline MRT, h & $19.2(27.4)$ & $15.0(27.9)$ & $1.28(1.21-1.35)$ & $<0.0001$ \\
\hline $\mathrm{CL}, \mathrm{dL} / \mathrm{h} / \mathrm{kg}$ & $0.021(28.5)$ & $0.030(31.0)$ & $0.67(0.64-0.71)$ & $<0.0001$ \\
\hline$V_{\mathrm{ss}}, \mathrm{dL} / \mathrm{kg}$ & $0.39(19.1)$ & $0.46(16.7)$ & $0.86(0.82-0.90)$ & $<0.0001$ \\
\hline
\end{tabular}

$A U C$ area under the curve, $A U C_{i n f}$ AUC from time zero to infinity, $A U C_{\text {last }}$ AUC from time zero to the last data point, $C I$ confidence interval, $C L$ total body clearance, $C_{\max }$ maximum concentration, $C V$ coefficient of variation, $M R T$ mean residence time, $N A$ not applicable, $r A H F-P F M$ antihemophilic factor (recombinant) plasma/albumin-free method, $t_{1 / 2}$ half-life, $V_{s s}$ volume of distribution at steady state

${ }^{\text {a }}$ Ratio of BAY 81-8973:rAHF-PFM

The popPK model was used in simulations to assess FVIII threshold levels following administration of BAY 81-8973 and rAHF-PFM in 1000 virtual patients. Time to reach threshold levels of $1,3,5$, and $10 \mathrm{IU} / \mathrm{dL}$ after a single infusion of BAY 81-8973 or rAHF-PFM was derived from simulated dense profiles. After single-dose administration, the median time to a FVIII level of $1 \mathrm{IU} / \mathrm{dL}$ was approximately $27 \%$ (approximately $18 \mathrm{~h}$ ) longer for BAY 81-8973 versus rAHF-PFM over a dose range of 25-50 IU/kg (Table 4). Similar results were obtained for 3, 5, and $10 \mathrm{IU} / \mathrm{dL}$ FVIII thresholds. Correspondingly, a substantially higher proportion of patients could maintain trough levels above threshold with the same dose of BAY 81-8973 versus rAHF-PFM (Table 5). Based on these 
Table 3 Parameter estimates of the population pharmacokinetic model

\begin{tabular}{llccl}
\hline Parameter & Units & Estimate & RSE $^{\mathrm{a}} \%$ & Description \\
\hline $\mathrm{CL}^{\mathrm{KOV}}$ & $\mathrm{dL} / \mathrm{h}$ & 1.51 & 7.33 & Clearance of BAY 81-8973 \\
$V 1^{\mathrm{KOV}, \mathrm{ADV}}$ & $\mathrm{dL}$ & 23.6 & 2.76 & Central volume of distribution of BAY 81-8973 and rAHF-PFM \\
$Q^{\mathrm{KOV}, \mathrm{ADV}}$ & $\mathrm{dL} / \mathrm{h}$ & 1.59 & 5.5 & Intercompartmental clearance of BAY 81-8973 and rAHF-PFM \\
$V 2^{\mathrm{KOV}}$ & $\mathrm{dL}$ & 5.35 & 12.8 & Peripheral volume of distribution of BAY 81-8973 \\
$\Delta \mathrm{CL}^{\mathrm{ADV}}$ & $\%$ & 47.8 & 8.05 & Change in clearance for rAHF-PFM compared with BAY 81-8973 \\
$\Delta V 2^{\mathrm{ADV}}$ & $\%$ & 23.4 & Change in peripheral volume of distribution for rAHF-PFM compared \\
& & & & with BAY 81-8973 \\
$\mathrm{IIV}$ & $\mathrm{CV} \%^{\mathrm{b}}$ & 27.2 & 13.9 & Intraindividual variability in clearance \\
$\mathrm{IIV}_{V_{1}}$ & $\mathrm{CV} \%^{\mathrm{b}}$ & 7.93 & 36.9 & Intraindividual variability in central volume of distribution \\
$\mathrm{IIV}_{\Delta \mathrm{Cl}}$ & $\mathrm{CV} \%^{\mathrm{b}}$ & 35 & 27.1 & Intraindividual variability in change in clearance \\
$\mathrm{IOV}_{F_{1}}$ & $\mathrm{CV} \%^{\mathrm{b}}$ & 10.3 & 31.7 & Intraoccasional variability in bioavailability \\
$\varepsilon_{\mathrm{prop}}$ & $\mathrm{CV} \%^{\mathrm{c}}$ & 5.73 & 6.84 & Proportional residual error \\
\hline
\end{tabular}

$C V$ coefficient of variation, $r A H F-P F M$ antihemophilic factor (recombinant) plasma/albumin-free method, $R S E$ relative standard error, $S Q R T$ square root

a Assessed via bootstrap ( $n=500$, with 485 successful runs)

b Calculated by SQRT(EXP(variance) -1$) \times 100$

c Calculated by SQRT(variance) $\times 100$

simulations, a higher dose of rAHF-PFM compared with BAY 81-8973 would be needed for $50 \%$ of patients to maintain FVIII levels $>1 \mathrm{IU} / \mathrm{kg}$ (161 vs. $52.4 \mathrm{IU} / \mathrm{kg}$ on a twice-weekly regimen; 39.1 vs. $14.4 \mathrm{IU} / \mathrm{kg}$ on a threetimes-weekly regimen), which equates to an approximately $72 \mathrm{IU} / \mathrm{kg}$ higher weekly dose for rAHF-PFM versus BAY 81-8973 (Table 6).

\section{Discussion}

This open-label, randomized study demonstrated improved PK properties of FVIII with BAY 81-8973 compared with rAHF-PFM following single doses. Mean $t_{1 / 2}$ was $24 \%$ and $16 \%$ longer for BAY 81-8973 compared with rAHF-PFM using the one-stage and chromogenic assays, respectively. A prolonged $t_{1 / 2}$ for BAY 81-8973 versus rAHF-PFM was observed in 16 of 18 patients, indicating that this was a consistent finding. The consistently high degree of sialic acid capping of $\mathrm{N}$-terminal glycans in BAY 81-8973 as a result of a refined manufacturing process [9] may have affected the $t_{1 / 2}$ of BAY 81-8973, as has been shown for some mammalian proteins [10]. In addition, $\mathrm{AUC}_{\text {inf }}$ was $32 \%$ and $48 \%$ higher in patients after infusion with BAY 81-8973 compared with rAHF-PFM using the one-stage and chromogenic assays, respectively. The difference in the PK parameters based on the two assays is not uncommon for FVIII products $[9,15]$. In general, the chromogenic assay has higher values for the same sample compared with the one-stage assay, which has been shown for many FVIII products. The popPK models for FVIII after administration of BAY 81-8973 and rAHF-PFM adequately describe the data from this study. The model parameters and results are consistent with a previous popPK analysis [18] and the PK data based on the noncompartmental analysis [9].

The comparison of BAY 81-8973 and rAHF-PFM PK in a single study, as has been done here, is advantageous because across-study comparisons of PK data, including $t_{1 / 2}$ values, for FVIII products may lead to discrepant and misleading conclusions. For example, reported $t_{1 / 2}$ for rFVIII-FS ranged from 12.0 to $13.7 \mathrm{~h}$ in two published studies and the US prescribing information $[9,19,20]$. In an Octapharma-sponsored study designed to show non-inferiority of simoctocog alfa (Nuwiq ${ }^{\circledR}$; Octapharma, Stockholm, Sweden) versus rFVIII-FS, $t_{1 / 2}$ for rFVIII-FS was 16.1 and $18.8 \mathrm{~h}$ using the chromogenic and one-stage assays, respectively [14]. For rAHF-PFM, $t_{1 / 2}$ ranged from 10.4 to $14.7 \mathrm{~h}$ across several studies [16, 21-24]. PK variability is influenced by several factors. For example, FVIII CL is highly correlated with von Willebrand factor [25]. Therefore, for an adequate comparison of PK characteristics of FVIII products, the products should be evaluated in the same population of patients.

Similar randomized, single-dose, crossover study designs have been used to compare PK parameters between different rFVIII products in other clinical studies. B-domain-deleted rFVIII (BDD-rFVIII) was found to be bioequivalent to rAHF-PFM based on AUC and $C_{\max }$ [26]. Turoctocog alfa was also found to be bioequivalent to rAHFPFM using AUC, $C_{\max }$, incremental recovery, $t_{1 / 2}$, and CL [27]. In both studies, $t_{1 / 2}$ was slightly shorter for BDD-rFVIII 

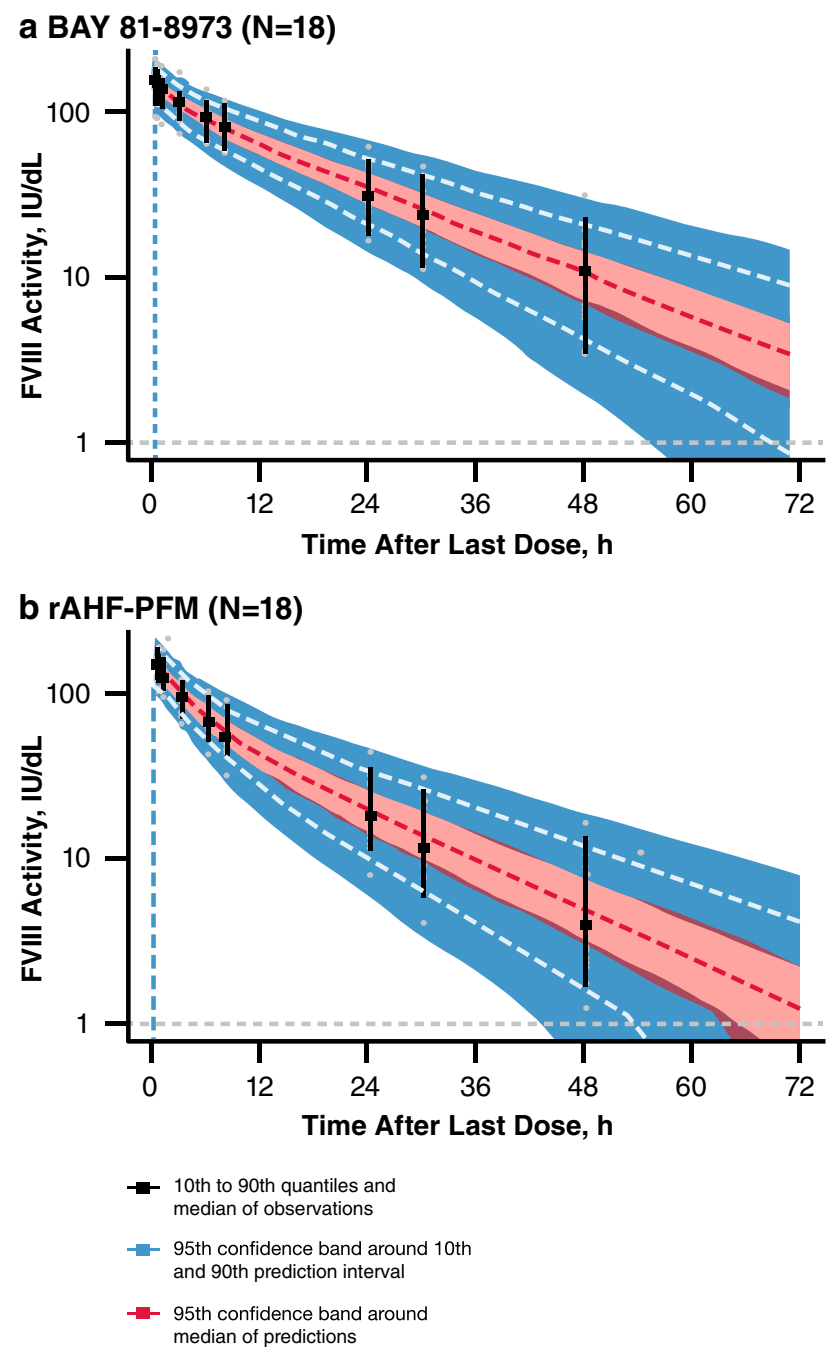

Fig. 2 FVIII level-time profiles in the population pharmacokinetic model for a BAY 81-8973 and b rAHF-PFM. FVIII factor VIII, rAHF-PFM antihemophilic factor (recombinant) plasma/albumin-free method

and turoctocog alfa versus rAHF-PFM [26, 27]. BAY 81-8973 was compared to its predecessor, rFVIII-FS, using a similar trial design; non-inferiority was demonstrated, and AUC and $t_{1 / 2}$ were increased $19 \%$ and $15 \%$, respectively, with BAY 81-8973 versus rFVIII-FS using the chromogenic assay (one-stage assay, 19\% and 10\% increase for AUC and $t_{1 / 2}$, respectively, vs. rFVIII-FS) [9].

Although several factors contribute to bleeding phenotype [3], maintenance of FVIII coagulant activity (FVIII:C) levels above threshold levels has been shown to prevent bleeding [8]. Because of the heterogeneity of bleeding phenotype and the high interpatient variability of PK parameters, the optimal threshold level of FVIII:C to prevent bleeding is likely to vary among patients [28, 29]. Nevertheless, FVIII products with favorable PK profiles may increase FVIII:C trough levels to provide additional protection against bleeding [30, 31]. In the current study,

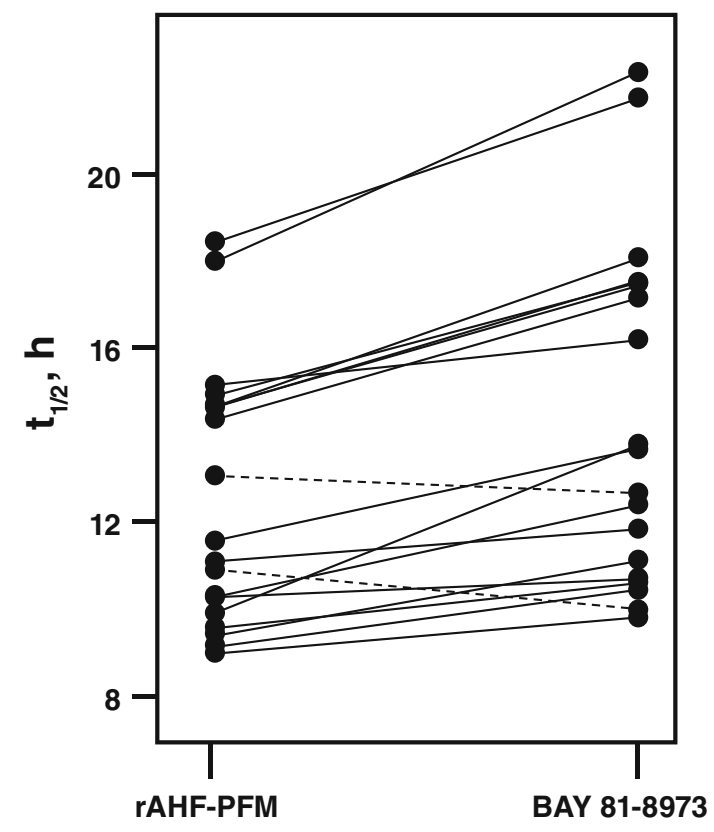

Fig. 3 Individual $t_{1 / 2}$ estimates following a dose of $30 \mathrm{IU} / \mathrm{kg}$ rAHFPFM and BAY 81-8973; a solid line indicates that a switch from rAHF-PFM to BAY 81-8973 leads to an expected increase in $t_{1 / 2}$ for this patient; a dashed line indicates a decrease in $t_{1 / 2}$ following a switch to BAY 81-8973. rAHF-PFM antihemophilic factor (recombinant) plasma/albumin-free method, $t_{1 / 2}$ half-life

simulations using the popPK model, which assumed equivalent efficacy of both products, showed that median time to a trough level of $1 \mathrm{IU} / \mathrm{dL}$ FVIII was longer for BAY 81-8973 versus rAHF-PFM, and that a lower dose of BAY 81-8973 compared with rAHF-PFM could achieve a threshold of $1 \mathrm{IU} / \mathrm{dL}$ FVIII. Furthermore, because increased trough levels are associated with bleeding protection in some patients $[8,28]$, increased trough levels for BAY 81-8973 versus rAHF-PFM at the same dose may provide improved bleeding protection with BAY 81-8973.

In some patients, prolonged $t_{1 / 2}$ and higher FVIII levels at various time points may allow for less frequent infusions while still maintaining desired threshold levels. Low annualized bleeding rates were reported in patients randomized to twice-weekly prophylaxis with BAY 81-8973 [12]. Although additional evidence is needed, low annualized bleeding rates may be the result of an improved PK profile for BAY 81-8973 compared with other FVIII products, such as rFVIII-FS [9] and rAHF-PFM (reported in this study).

A potential limitation of this study was the need to simulate trough levels at 72 and $96 \mathrm{~h}$ because samples were only collected up to $48 \mathrm{~h}$, which is typical for the assessment of PK characteristics of FVIII molecules [9, 26, 27]. However, this potential limitation does not preclude predictions of trough levels at 72 and $96 \mathrm{~h}$ because the PK of FVIII molecules are well characterized and the popPK model provided an accurate description of the data. 
Fig. 4 Pharmacokinetic trough level at steady state following a dose of $30 \mathrm{IU} / \mathrm{kg}$ rAHF-PFM and BAY 81-8973; a solid line indicates that a switch from rAHF-PFM to BAY 81-8973 leads to an expected increase in trough levels for this patient; a dashed line indicates a decrease in trough level following a switch to BAY 81-8973. $C_{\text {trough }}$ trough level, rAHF-PFM antihemophilic factor (recombinant) plasma/albuminfree method

Fig. 5 Simulation of a typical steady-state profile of a switch from $30 \mathrm{IU} / \mathrm{kg}$ rAHF-PFM to $30 \mathrm{IU} / \mathrm{kg}$ BAY 81-8973 a twice weekly and $\mathbf{b}$ three times weekly. Shaded areas indicate times when FVIII

concentrations are $<1 \mathrm{IU} / \mathrm{dL}$ for panel $\mathbf{a}$ and $<3 \mathrm{IU} / \mathrm{dL}$ for panel b. FVIII factor VIII, $r A H F-P F M$ antihemophilic factor (recombinant) plasma/albuminfree method

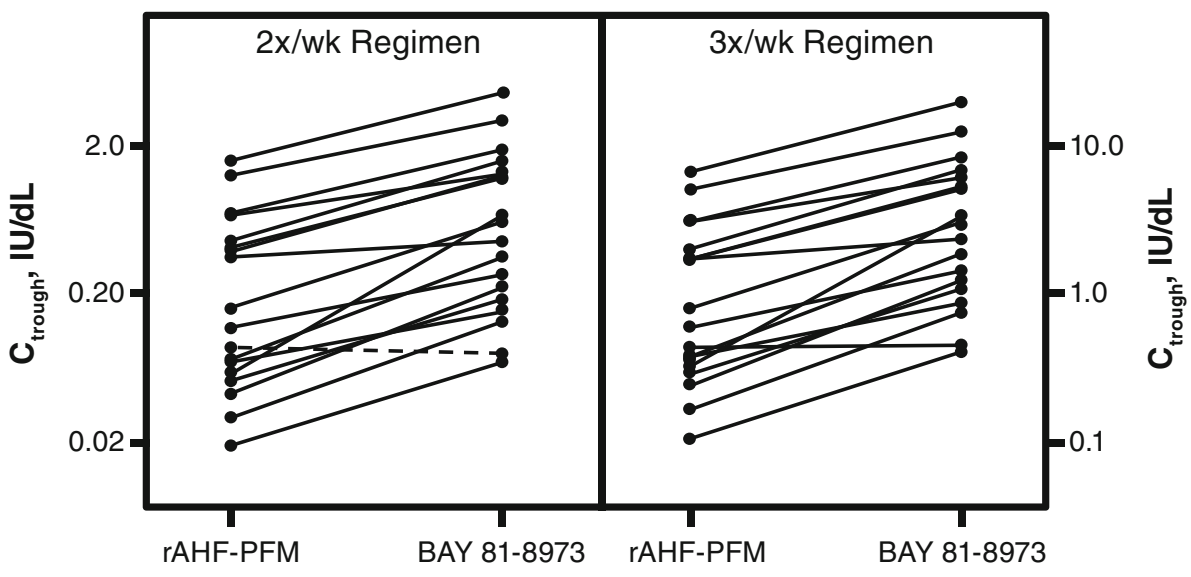

a 30 IU/kg 2x/wk

rAHF-PFM (steady state)
Switch to BAY 81-8973

$=$ = " Stay on rAHF-PFM

Time below <1 IU/dL:

rAHF-PFM

BAY 81-8973

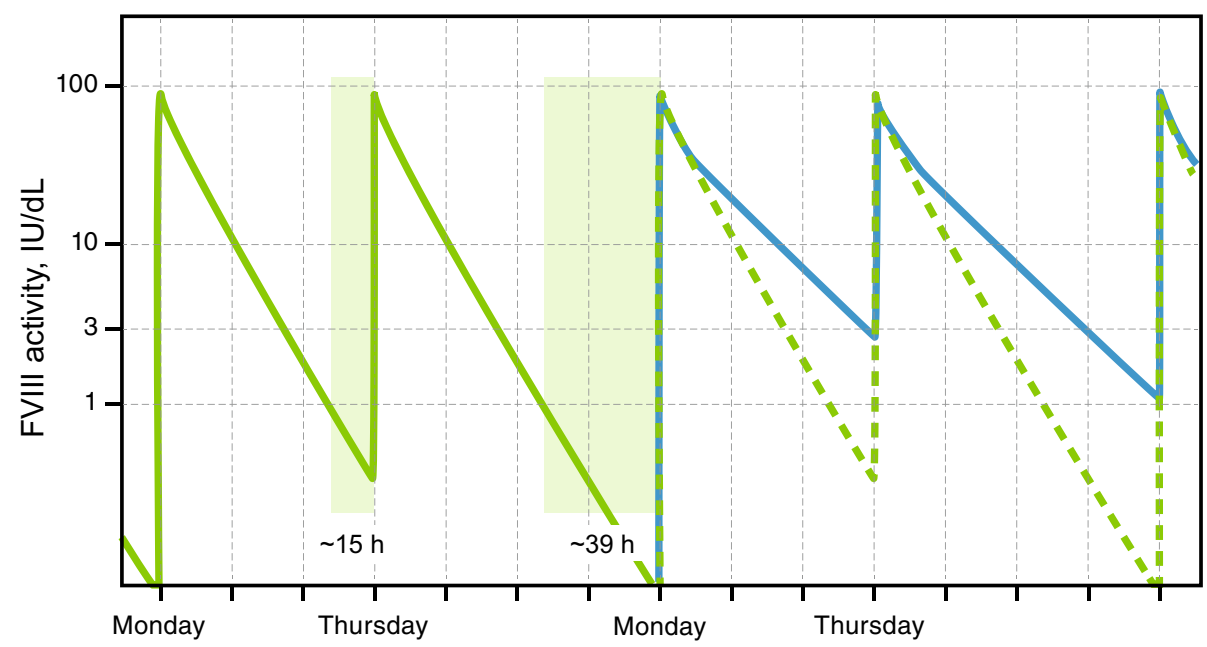

b $30 \mathrm{lU} / \mathrm{kg} 3 \mathrm{x} / \mathrm{wk}$

rAHF-PFM (steady state)
Switch to BAY 81-8973

- = " Stay on rAHF-PFM

Time below <3 IU/dL:

rAHF-PFM

BAY 81-8973

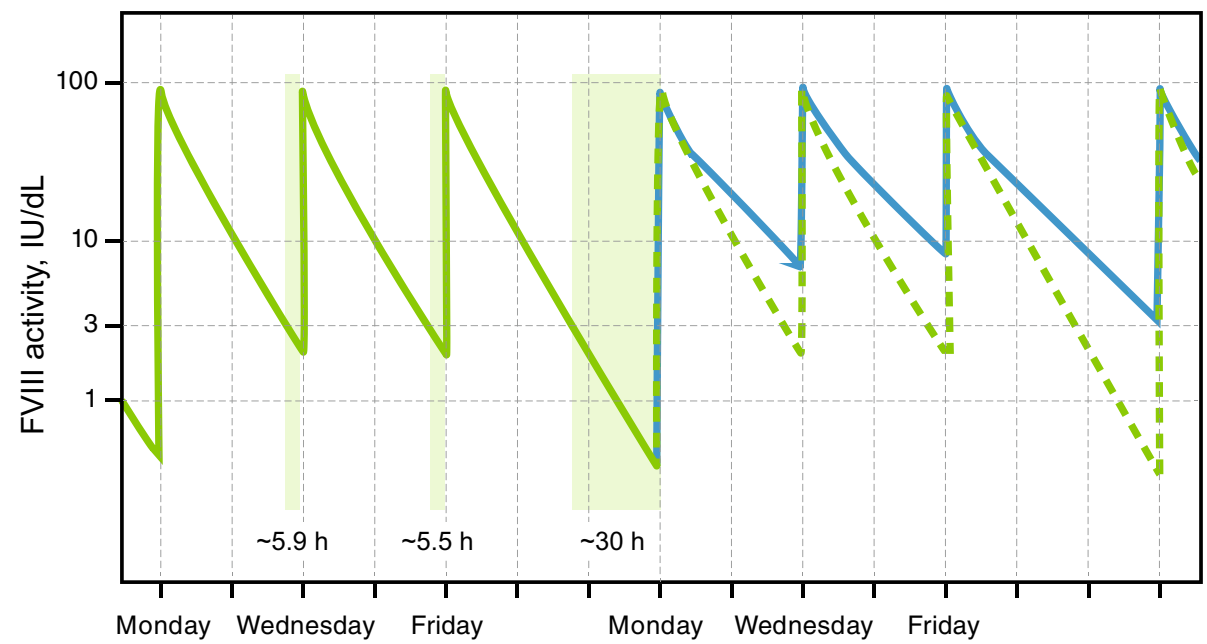


Table 4 Median time to FVIII threshold level after a single intravenous dose (simulation of 1000 patients)

\begin{tabular}{|c|c|c|c|c|c|}
\hline $\begin{array}{l}\text { Dose, } \\
\text { IU/kg }\end{array}$ & $\begin{array}{l}\text { Threshold level, } \\
\text { IU/dL }\end{array}$ & $\begin{array}{l}\text { BAY } 81-8973, \mathrm{~h} \text { (5th and } \\
\text { 95th Percentiles) }\end{array}$ & $\begin{array}{l}\text { rAHF-PFM, } h \text { (5th and } \\
\text { 95th Percentiles) }\end{array}$ & Ratio $^{a}$ & Difference $^{\mathrm{b}}, \mathrm{h}$ \\
\hline 25 & 1 & $80.5(50.5 ; 122.5)$ & $62.5(39.5 ; 100)$ & 1.29 & 18 \\
\hline 25 & 3 & $59(36.5 ; 90)$ & $43.5(26.5 ; 71)$ & 1.36 & 15.5 \\
\hline 25 & 5 & $49(30 ; 74.5)$ & $34.5(24 ; 57)$ & 1.42 & 14.5 \\
\hline 25 & 10 & $34.5(24 ; 53)$ & $24(15 ; 39)$ & 1.44 & 10.5 \\
\hline 30 & 1 & $84.5(53 ; 128)$ & $65.5(42 ; 104.5)$ & 1.29 & 19 \\
\hline 30 & 3 & $63(38.5 ; 95.5)$ & $46.5(28.5 ; 75.5)$ & 1.35 & 16.5 \\
\hline 30 & 5 & $52.5(32.5 ; 80)$ & $38(24 ; 62)$ & 1.38 & 14.5 \\
\hline 30 & 10 & $38.5(24 ; 58.5)$ & $26(15 ; 43.5)$ & 1.48 & 12.5 \\
\hline 40 & 1 & $90(56.5 ; 136.5)$ & $71(45.5 ; 112.5)$ & 1.27 & 19 \\
\hline 40 & 3 & $68.5(42.5 ; 104)$ & $51.5(32 ; 83.5)$ & 1.33 & 17 \\
\hline 40 & 5 & $58.5(36 ; 89)$ & $43(26 ; 70)$ & 1.36 & 15.5 \\
\hline 40 & 10 & $44.5(27 ; 67.5)$ & $31(24 ; 51)$ & 1.44 & 13.5 \\
\hline 50 & 1 & $94.5(59.5 ; 143.5)$ & $74.5(48 ; 118.5)$ & 1.27 & 20 \\
\hline 50 & 3 & $73(45 ; 110.5)$ & $55.5(34.5 ; 89)$ & 1.32 & 17.5 \\
\hline 50 & 5 & $63(38.5 ; 95.5)$ & $46.5(28.5 ; 75.5)$ & 1.35 & 16.5 \\
\hline 50 & 10 & $49(30 ; 74.5)$ & $34.5(24 ; 57)$ & 1.42 & 14.5 \\
\hline
\end{tabular}

FVIII factor VIII, rAHF-PFM antihemophilic factor (recombinant) plasma/albumin-free method

a Ratio of BAY 81-8973:rAHF-PFM

b Difference of BAY 81-8973 - rAHF-PFM

Table 5 Proportion of patients maintaining FVIII levels above the threshold (simulation of 1000 patients)

\begin{tabular}{|c|c|c|c|c|c|c|c|}
\hline \multirow{2}{*}{$\begin{array}{l}\text { Dose, } \\
\text { IU/kg }\end{array}$} & \multirow{2}{*}{$\begin{array}{l}\text { Threshold, } \\
\text { IU/dL }\end{array}$} & \multicolumn{3}{|c|}{$2 \times /$ week regimen $^{a}$} & \multicolumn{3}{|c|}{$3 \times /$ week regimen $^{\mathrm{b}}$} \\
\hline & & $\begin{array}{l}\text { BAY } 81-8973 \\
\text { patients, \% }\end{array}$ & $\begin{array}{l}\text { rAHF-PFM } \\
\text { patients, \% }\end{array}$ & Ratio $^{c}$ & $\begin{array}{l}\text { BAY 81-8973 } \\
\text { patients, } \%\end{array}$ & $\begin{array}{l}\text { rAHF-PFM } \\
\text { patients, \% }\end{array}$ & Ratio $^{c}$ \\
\hline 25 & 1 & 27.0 & 6.9 & 3.91 & 69.1 & 34.5 & 2.00 \\
\hline 25 & 3 & 4.0 & 0.5 & 8.00 & 28.1 & 6.8 & 4.13 \\
\hline 25 & 5 & 1.1 & 0 & - & 11.0 & 2.2 & 5.00 \\
\hline 30 & 1 & 33.6 & 10.0 & 3.36 & 75.1 & 40.1 & 1.87 \\
\hline 30 & 3 & 6.2 & 1.1 & 5.64 & 34.7 & 10.0 & 3.47 \\
\hline 30 & 5 & 2.4 & 0.1 & 24.0 & 15.2 & 3.3 & 4.61 \\
\hline 40 & 1 & 42.2 & 14.0 & 3.01 & 81.9 & 50.9 & 1.61 \\
\hline 40 & 3 & 10.4 & 2.4 & 4.33 & 46.6 & 15.1 & 3.09 \\
\hline 40 & 5 & 3.8 & 0.5 & 7.60 & 26.4 & 5.8 & 4.55 \\
\hline 50 & 1 & 49.2 & 18.4 & 2.67 & 85.6 & 58.2 & 1.47 \\
\hline 50 & 3 & 15.3 & 3.5 & 4.37 & 55.2 & 20.4 & 2.71 \\
\hline 50 & 5 & 6.2 & 1.1 & 5.64 & 34.7 & 10.0 & 3.47 \\
\hline
\end{tabular}

FVIII factor VIII, rAHF-PFM antihemophilic factor (recombinant) plasma/albumin-free method

${ }^{a}$ Doses on Monday and Thursday

b Doses on Monday, Wednesday, and Friday

${ }^{c}$ Ratio of BAY 81-8973:rAHF-PFM 
Table 6 Dosing required to maintain FVIII plasma levels $>1$ IU/dL (simulation of 1000 patients)

\begin{tabular}{|c|c|c|c|c|c|}
\hline \multirow[t]{2}{*}{ Dosing frequency } & \multicolumn{2}{|l|}{ BAY $81-8973$} & \multicolumn{2}{|l|}{ rAHF-PFM } & \multirow[t]{2}{*}{ Ratio $^{\mathrm{a}}$} \\
\hline & $\begin{array}{l}\text { Dose for } 50 \% \text { of patients to } \\
\text { maintain }>1 \mathrm{IU} / \mathrm{dL}, \mathrm{IU} / \mathrm{kg}\end{array}$ & $\begin{array}{l}\text { Absolute dose per year } \\
\text { for } 70-\mathrm{kg} \text { patient, IU }\end{array}$ & $\begin{array}{l}\text { Dose for } 50 \% \text { of patients to } \\
\text { maintain }>1 \mathrm{IU} / \mathrm{dL}, \mathrm{IU} / \mathrm{kg}\end{array}$ & $\begin{array}{l}\text { Absolute dose per year } \\
\text { for } 70-\mathrm{kg} \text { patient, IU }\end{array}$ & \\
\hline $2 \times /$ week & 52.4 & 381,472 & 161 & $1,172,080$ & 0.33 \\
\hline $3 \times /$ week & 14.4 & 157,248 & 39.1 & 426,972 & 0.37 \\
\hline
\end{tabular}

FVIII factor VIII, $r A H F-P F M$ antihemophilic factor (recombinant) plasma/albumin-free method

${ }^{\text {a }}$ Ratio of BAY 81-8973:rAHF-PFM

\section{Conclusions}

BAY 81-8973 showed superior PK properties compared with rAHF-PFM following a single infusion in patients with severe hemophilia. Simulations based on popPK modeling showed that a substantially higher proportion of patients could be maintained at trough levels above threshold with BAY 81-8973 compared with rAHF-PFM. A threshold of $>1$ IU/dL FVIII may be achieved with a lower dose of BAY 81-8973 compared with rAHF-PFM, and median time to $1 \mathrm{IU} / \mathrm{dL}$ FVIII was longer for BAY 81-8973 versus rAHF-PFM.

Acknowledgements The authors thank the following people from Bayer: Sue Cheng (medical expert), Maxim Sapozhnikov (statistical programmer), Heinz Delesen (statistician), and Katharina Sommers (statistician).

\section{Compliance with Ethical Standards}

Funding This study was funded by Bayer Pharma AG, Leverkusen, Germany. Medical writing assistance was provided by Ken Wannemacher, $\mathrm{PhD}$, from Complete Healthcare Communications, LLC (Chadds Ford, PA, USA) and was funded by Bayer. Open access publication of this manuscript was sponsored by Bayer.

Conflict of interest Anita Shah is an employee of Bayer, and Alexander Solms, Dirk Garmann, and Yvonne Katterle are employees of Bayer Pharma AG. Toshko Lissitchkov, Verzhiniya Avramova, and Stanislav Simeonov have received Grant/research support for clinical trial participation from Bayer.

Ethical approval The procedures performed in this study were in accordance with good clinical practice guidelines and the guiding principles detailed in the Declaration of Helsinki.

Informed consent Informed consent was provided by the patients, and the protocol was approved by the site's independent Ethics Committee/Institutional Review Board (Local Ethics Committee at SHATHD-Sofia, 6, Plovdivsko pole Str., 1756 Sofia, Bulgaria).

Open Access This article is distributed under the terms of the Creative Commons Attribution-NonCommercial 4.0 International License (http://creativecommons.org/licenses/by-nc/4.0/), which permits any noncommercial use, distribution, and reproduction in any medium, provided you give appropriate credit to the original author(s) and the source, provide a link to the Creative Commons license, and indicate if changes were made.

\section{References}

1. Srivastava A, Brewer AK, Mauser-Bunschoten EP, et al. Guidelines for the management of hemophilia. Haemophilia. 2013;19(1):e1-47.

2. National Hemophilia Foundation. Medical and Scientific Advisory Council (MASAC) recommendations concerning prophylaxis (regular administration of clotting factor concentrate to prevent bleeding). Document \#241. 2016. https://www. hemophilia.org/sites/default/files/document/files/241Prophylaxis. pdf. Accessed 25 May 2016.

3. Pavlova A, Oldenburg J. Defining severity of hemophilia: more than factor levels. Semin Thromb Hemost. 2013;39(7):702-10.

4. Rendo P, Shafer F, Korth-Bradley JM, et al. Factors that influence the bleeding phenotype in severe hemophilic patients. Blood Coagul Fibrinolysis. 2013;24(7):683-90.

5. Valentino LA. Considerations in individualizing prophylaxis in patients with haemophilia A. Haemophilia. 2014;20(5):607-15.

6. Collins PW. Personalized prophylaxis. Haemophilia. 2012;18 (Suppl 4):131-5.

7. Ahnstrom J, Berntorp E, Lindvall K, et al. A 6-year follow-up of dosing, coagulation factor levels and bleedings in relation to joint status in the prophylactic treatment of haemophilia. Haemophilia. 2004;10(6):689-97.

8. Collins PW, Blanchette VS, Fischer K, et al. Break-through bleeding in relation to predicted factor VIII levels in patients receiving prophylactic treatment for severe hemophilia A. J Thromb Haemost. 2009; 7(3):413-20.

9. Shah A, Delesen H, Garger S, et al. Pharmacokinetic properties of BAY 81-8973, a full-length recombinant factor VIII. Haemophilia. 2015;21(6):766-71.

10. Bovenschen N, Rijken DC, Havekes LM, et al. The B domain of coagulation factor VIII interacts with the asialoglycoprotein receptor. J Thromb Haemost. 2005;3(6):1257-65.

11. Saxena K, Lalezari S, Oldenberg J, et al. Efficacy and safety of BAY 81-8973, a full-length recombinant factor VIII: results from the LEOPOLD I trial. Haemophilia. 2016;22(5):706-12.

12. Kavakli K, Yang R, Rusen L, et al. Prophylaxis versus ondemand treatment with BAY 81-8973, a full-length plasma-proteinfree rFVIII product: results from a randomized trial (LEOPOLD II). J Thromb Haemost. 2015;13(3):360-9.

13. Ljung R, Kenet G, Mancuso ME, et al. BAY 81-8973 safety and efficacy for prophylaxis and treatment of bleeds in previously treated children with severe haemophilia A: results of the LEOPOLD Kids Trial. Haemophilia. 2016;22(3):354-60.

14. Lissitchkov T, Tiede A, Valentino L, et al. Pharmacokinetics and efficacy of on-demand treatment with human-cl rhFVIII in previously treated patients with severe hemophilia A [abstract]. Haemophilia. 2012;18(Suppl 3):150.

15. Jimenez-Yuste $\mathrm{V}$, Lejniece $\mathrm{S}$, Klamroth $\mathrm{R}$, et al. The pharmacokinetics of a B-domain truncated recombinant factor VIII, 
turoctocog alfa $\left(\right.$ NovoEight $\left.^{\circledR}\right)$, in patients with hemophilia A. J Thromb Haemost. 2015;13(3):370-9.

16. Konkle BA, Stasyshyn O, Chowdary P, et al. Pegylated, fulllength, recombinant factor VIII for prophylactic and on-demand treatment of severe hemophilia A. Blood. 2015;126(9):1078-85.

17. Klamroth R, Simpson M, von Depka-Prondzinski M, et al. Comparative pharmacokinetics of rVIII-single chain and octocog alfa $\left(\right.$ Advate $\left.^{\circledR}\right)$ in patients with severe haemophilia A. Haemophilia. 2016;22(5):730-8.

18. Bjorkman S, Oh M, Spotts G, et al. Population pharmacokinetics of recombinant factor VIII: the relationships of pharmacokinetics to age and body weight. Blood. 2012;119(2):612-8.

19. Kogenate ${ }^{\circledR}$ FS (antihemophilic factor [recombinant] formulated with sucrose). Full prescribing information. Whippany: Bayer; 2015.

20. Coyle TE, Reding MT, Lin JC, et al. Phase I study of BAY 94-9027, a PEGylated B-domain-deleted recombinant factor VIII with an extended half-life, in subjects with hemophilia A. J Thromb Haemost. 2014;12(4):488-96.

21. Powell JS, Josephson NC, Quon D, et al. Safety and prolonged activity of recombinant factor VIII Fc fusion protein in hemophilia A patients. Blood. 2012;119(13):3031-7.

22. Valentino LA, Mamonov V, Hellmann A, et al. A randomized comparison of two prophylaxis regimens and a paired comparison of on-demand and prophylaxis treatments in hemophilia A management. J Thromb Haemost. 2012;10(3):359-67.

23. Tarantino MD, Collins PW, Hay CR, et al. Clinical evaluation of an advanced category antihaemophilic factor prepared using a plasma/albumin-free method: pharmacokinetics, efficacy, and safety in previously treated patients with haemophilia A. Haemophilia. 2004;10(5):428-37.
24. Advate ${ }^{\circledR}$ (antihemophilic factor [recombinant] plasma/albuminfree method). Full prescribing information. Westlake Village: Baxter Healthcare Corporation; 2014.

25. Lalezari S, Martinowitz U, Windyga J, et al. Correlation between endogenous VWF: Ag and PK parameters and bleeding frequency in severe haemophilia A subjects during three-timesweekly prophylaxis with rFVIII-FS. Haemophilia. 2014;20(1): e15-22.

26. Di Paola J, Smith MP, Klamroth R, et al. ReFacto and Advate: a single-dose, randomized, two-period crossover pharmacokinetics study in subjects with haemophilia A. Haemophilia. 2007;13(2): 124-30.

27. Martinowitz U, Bjerre J, Brand B, et al. Bioequivalence between two serum-free recombinant factor VIII preparations (N8 and ADVATE $^{\circledR}$ )-n open-label, sequential dosing pharmacokinetic study in patients with severe haemophilia A. Haemophilia. 2011;17(6):854-9.

28. Collins PW, Fischer K, Morfini M, et al. Implications of coagulation factor VIII and IX pharmacokinetics in the prophylactic treatment of haemophilia. Haemophilia. 2011;17(1):2-10.

29. Jayandharan GR, Srivastava A. The phenotypic heterogeneity of severe hemophilia. Semin Thromb Hemost. 2008;34(1):128-41.

30. Mahdi AJ, Obaji SG, Collins PW. Role of enhanced half-life factor VIII and IX in the treatment of haemophilia. Br J Haematol. 2015;169(6):768-76.

31. Collins PW, Bjorkman S, Fischer K, et al. Factor VIII requirement to maintain a target plasma level in the prophylactic treatment of severe hemophilia A: influences of variance in pharmacokinetics and treatment regimens. J Thromb Haemost. 2010;8(2):269-75. 